\section{Protective effects of antimalarials in Chinese patients with systemic lupus erythematosus}

We read with great interest the article by Müller-Calleja $e t ~ a l^{1}$ in which a novel mechanism of hydroxychloroquine (HCQ) was discovered, which might explain well-established anti-inflammatory effects of antimalarials. ${ }^{2-4}$ HCQ and chloroquine (CQ), both known as antimalarial drugs, have become fundamental therapeutic elements in systemic lupus erythematosus (SLE) in these decades. However, their specific benefits on organ involvement and long-term outcome remain to be elucidated.

To examine the role of antimalarials in different clinical aspects of Chinese patients with SLE, we retrieved the medical records of 1372 patients with SLE who experienced their first hospitalisation at 26 centres across Jiangsu, an eastern province of China, between January 1999 and December 2009 from a longitudinal SLE database collected by the Jiangsu Lupus Collaborative Group. ${ }^{5}$ Demographics of the enrolled patients are summarised in table 1. All the patients were followed up in 2015 to check for their survival status. Based on Kaplan-Meier survival analysis, the survival proportions for antimalarial users were 92.6\%, 90.3\% and $87.8 \%$ at 5,10 and 16 years, significantly higher than that of non-users $(83.1 \%, 78.9 \%$ and $76.0 \%, p=0.000)$ (figure $1 \mathrm{~A}$ ), which was consistent with previous reports in Latin American, African-American and Caucasian patients with SLE. ${ }^{6-8}$ There was no difference in survival proportions between HCQ users and CQ users.

In previous studies, the protective role of antimalarials was mainly concentrated on lupus nephritis. ${ }^{9}$ To further clarify this question, we performed univariate Cox proportional hazards regression analysis according to antimalarial exposure status on various clinical features. As shown in figure 1B, antimalarial drugs could exert their protective roles on a wide range of aspects, including but not limited to female gender, longer disease duration, a variety of organ involvement, hypocomplementemia and administration of glucocorticoids as well as cyclophosphamide. Besides renal involvement, patients with cardiopulmonary, gastrointestinal or haematological impairments also benefited from antimalarial treatment (all $p<0.0001$ by KaplanMeier survival analysis, figure 1C). Since antimalarials could

Table 1 Baseline characteristics of the patients with systemic lupus erythematosus by usage of antimalarials

\begin{tabular}{|c|c|c|c|}
\hline \multirow[b]{2}{*}{ Characteristic } & \multicolumn{2}{|c|}{ Antimalarial use } & \multirow[b]{2}{*}{$P$ values } \\
\hline & User & Non-user & \\
\hline \multicolumn{4}{|l|}{ Demographics } \\
\hline Numbers of patients & $562(41.0 \%)$ & $810(59.0 \%)$ & \\
\hline Women & $526(93.6 \%)$ & $740(91.4 \%)$ & ns \\
\hline Age (years) & $32.7(12.1)$ & $36.1(12.6)$ & $<0.0001$ \\
\hline \multicolumn{4}{|l|}{ Disease characteristics } \\
\hline Disease duration (years) & $3.1(5.0)$ & $3.7(5.4)$ & ns \\
\hline SLEDAI on admission & $14.4(7.4)$ & $14.6(8.8)$ & ns \\
\hline SDI $(\geq 1)$ on admission & $1.4(0.61)$ & $1.6(1.0)$ & ns \\
\hline \multicolumn{4}{|l|}{ Organ involvements $^{\dagger}$} \\
\hline Mucocutaneous & $420(74.7 \%)$ & $494(61.0 \%)$ & $<0.0001$ \\
\hline Neuropsychiatric & $26(4.6 \%)$ & $66(8.1 \%)$ & 0.0113 \\
\hline Musculoskeletal & $325(57.8 \%)$ & $419(51.7 \%)$ & 0.0276 \\
\hline Cardiopulmonary & $91(16.2 \%)$ & $191(23.6 \%)$ & 0.0009 \\
\hline Gastrointestinal & $114(20.2 \%)$ & $156(19.3 \%)$ & ns \\
\hline Ocular & $5(0.9 \%)$ & $4(0.5 \%)$ & ns \\
\hline Renal & $270(48.0 \%)$ & $431(53.2 \%)$ & ns \\
\hline Haematological & $234(41.6 \%)$ & $383(47.3 \%)$ & 0.0412 \\
\hline \multicolumn{4}{|l|}{ Serology } \\
\hline Anti-dsDNA positive & $265(50.4 \%)$ & $376(54.7 \%)$ & ns \\
\hline Anti-Sm positive & $164(32.6 \%)$ & $239(34.3 \%)$ & ns \\
\hline Anti-cardiolipin positive & $69(29.2 \%)$ & $77(29.7 \%)$ & ns \\
\hline RF positive & $112(30.0 \%)$ & $131(27.7 \%)$ & ns \\
\hline Low C3 complementł & $395(76.6 \%)$ & $489(72.4 \%)$ & ns \\
\hline Low C4 complementł & $343(76.6 \%)$ & $427(76.8 \%)$ & ns \\
\hline \multicolumn{4}{|l|}{ Medications } \\
\hline Glucocorticoids & $541(96.3 \%)$ & $723(89.3 \%)$ & $<0.0001$ \\
\hline Cyclophosphamide & $224(40.0 \%)$ & $358(44.2 \%)$ & ns \\
\hline Other immunosuppressants & $104(18.5 \%)$ & $129(15.9 \%)$ & ns \\
\hline \multicolumn{4}{|l|}{ Comorbidities } \\
\hline Hypertension & $9(1.6 \%)$ & $22(2.7 \%)$ & ns \\
\hline Diabetes mellitus & $26(4.6 \%)$ & $39(4.8 \%)$ & ns \\
\hline Infection & $15(2.7 \%)$ & $22(2.7 \%)$ & ns \\
\hline
\end{tabular}

*From the date of symptom onset to the date of first admission.

tWere assessed as Feng et $a t^{5}$ described.

¥Complement C3<0.8g/L, complement C4 $<0.2 \mathrm{~g} / \mathrm{L}$.

ns, non-significant; RF, rheumatoid factor; SDI, Systemic Lupus International Collaborating and Clinics /American College of Rheumatology Damage Index ; SLEDAI, Systemic Lupus Erythematosus Disease Activity Index. 
A

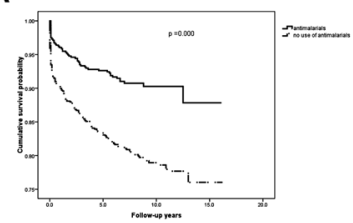

B

C
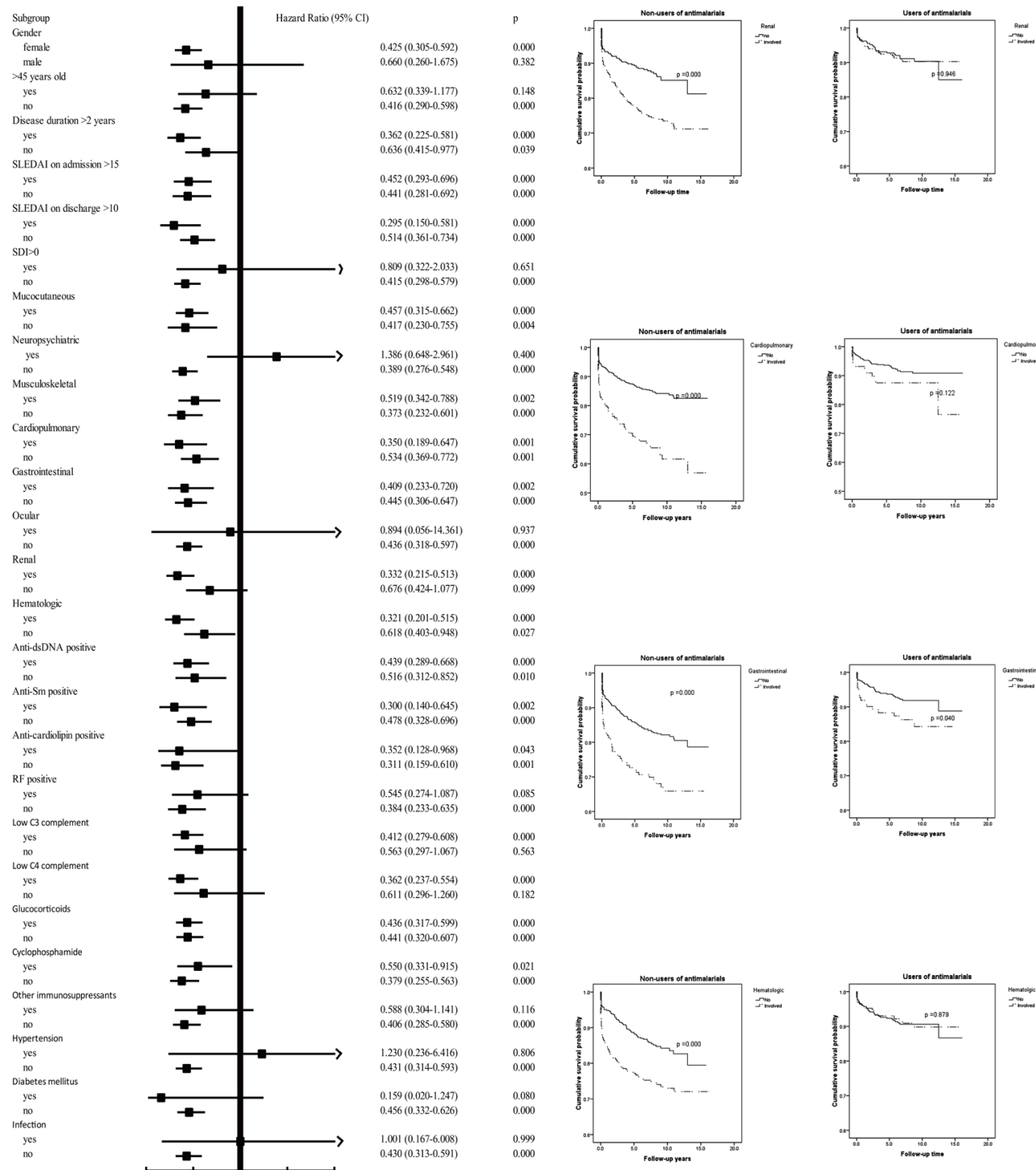

$0.295(0.150-0.581$

$0.514(0.361 .0 .734$

$0.809(0.322-2.033)$
$0415(0298-0.0599)$

$0.457(0.315-0.062$

$0.457(0.315-0.062)$
$0.417(0.230-0.755)$

$0.417(0.230-0.755)$
$1.386(0.648-2.961$

$1.386(0.648-2.961)$
$0.389(0.276-0.548)$

0.519 (0.342-0.788

$0.373(0.232-0.60)$

$0.350(0.189-0.647)$
$0.534(0.369-0.772)$

$0.409(0.233-0.720$

$0.445(0.306-0.647)$

$0.894(0.056-14.361)$

$0.332(0.215 .05 .513)$
$0.676(0.4241 .077)$

$0.676(0.4241 .077)$

$0.6118(0.2010 .0 .515$

$0.439(0.289-0.668$

0.439
$0.516(0.289-0.0612-0.858$

$0.300(0.140-0.645)$

$0.352(0.128 .0 .968$

$0.352(0.128 .0 .968)$
$0.311(0.159-0.610)$

$0.545(0.274 .1 .087)$

$0.545(0.2741 .087)$
$0.384(0.233-0.635)$

$0.412(0.279-0.608$

$0.563(0.297-1.067)$

$0.362(0.237-0.554)$

$0.611(0.296-1.260)$

$0.436(0.317-0.599$

0.441 (0.320-0.607)

$0.550(0.331-0.915)$
$0.379(0.255-0.563)$

$0.588(0.304-1.141$

$0.406(0.285-0.580$

$1.230(0.236-6.416)$

$0.159(0.020-1.247)$

$0.159(0.020-1.247)$
$0.456(0.332-0.626$

$0.456(0.332-0.626$

$1.001(0.167-6.008)$
$0.430(0.313-0.591)$
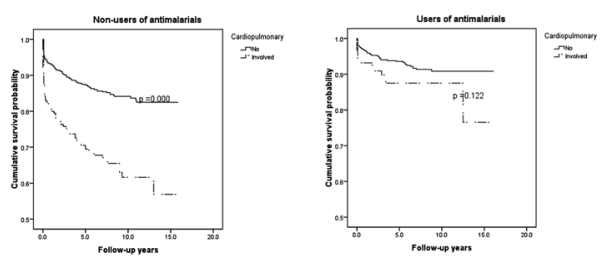

0.000
0.000
0.099

0.09

0.000

0.000
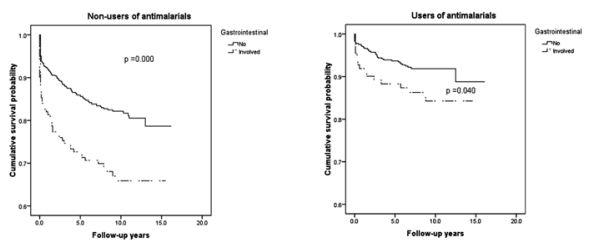

0.000

0.000

0.000

0.021

0.000
0.116
0.000
0.806
0.000
0.080
0.000
0.999
0.000
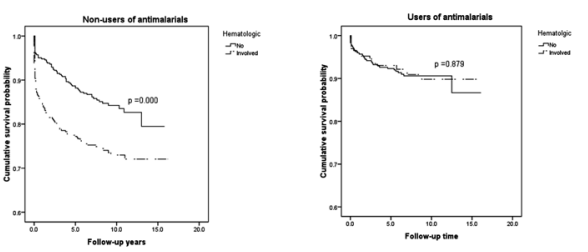

Figure 1 (A) Survival curves of patients with systemic lupus erythematosus (SLE) divided by whether using antimalarial drugs. (B) Examinations of HRs of SLE patients' characters in using antimalarials. HRs are shown with $95 \% \mathrm{Cl}$. (C) Comparisons of survival according to SLE patients' organ involvements and antimalarial usage. SDI, Systemic Lupus International Collaborating and Clinics /American College of Rheumatology Damage Index ; SLEDAI, Systemic Lupus Erythematosus Disease Activity Index.

interference with the assembly of endosomal NADPH oxidase to inhibit the overactivity of immune system, ${ }^{1}$ users were expected to have better outcomes than non-users when they were accompanied with vital organ involvements.

The effect of antimalarials on alleviating organ damage may also be related to lipid lowering, ${ }^{2}$ glycaemic controlling ${ }^{3}$ and thrombosis prevention. ${ }^{4}$ In this cohort, 376 patients had the record of second hospitalisation before 2015, of which 165 received antimalarial treatment. Blood level of total cholesterol was lower in antimalarial treating group than the non-treated group $(4.47 \pm 1.25 \mathrm{mmol} / \mathrm{L}$ vs $5.04 \pm 2.17 \mathrm{mmol} / \mathrm{L}, \mathrm{p}<0.05)$, and there was no difference between the two groups in the first hospitalisation. Other metabolic data, such as systolic and diastolic blood pressure, fasting blood sugar, triglyceride and uric acid, were similar between the two groups in two times of hospitalisation. Although percentages of leucopenia, anaemia and thrombocytopenia were similar between antimalarial users and non-users during the first hospitalisation, non-users were more likely to be anaemia on their second inpatients visits $(46.5 \%$ vs $58.2 \%, \mathrm{p}<0.05)$. 
In conclusion, our data show that the use of antimalarials could improve long-term outcome of Chinese patients with SLE. These drugs possess protective effects on a wide range of clinical aspects. Patients with vital organ involvements, especially cardiopulmonary, gastrointestinal, renal or haematological impairments, may gain more benefits from antimalarial treatments.

Fan Wang, ${ }^{1}$ Wei Zhang, ${ }^{2,3}$ Shiying Wang, ${ }^{1}$ Wenyou Pan, ${ }^{4}$ Lin Liu, ${ }^{5}$ Min Wu, ${ }^{6}$ Fuwan Ding, ${ }^{7}$ Huaixia Hu, ${ }^{8}$ Xiang Ding, ${ }^{9}$ Hua Wei, ${ }^{10}$ Yaohong Zou, ${ }^{11}$ Xian Qian, ${ }^{12}$ Meimei Wang, ${ }^{13}$ Jian Wu, ${ }^{14}$ Juan Tao, ${ }^{15}$ Jun Tan, ${ }^{16}$ Zhanyun Da, ${ }^{17}$ Miaojia Zhang, ${ }^{18}$ Jing Li, ${ }^{19}$ Xuebing Feng, ${ }^{1}$ Lingyun Sun ${ }^{1}$

${ }^{1}$ Department of Rheumatology and Immunology, The Affiliated Drum Tower Hospital of Nanjing University Medical School, Nanjing, China

${ }^{2}$ Department of Rheumatology and Immunology, The Affiliated Drum Tower Clinical Medical School, Nanjing Medical University, Nanjing, China

${ }^{3}$ Department of Rheumatology, Nanjing Medical University Affiliated Jiangning Hospital, Nanjing, China

${ }^{4}$ Department of Rheumatology, Huaian First People's Hospital, Huaian, China

${ }^{5}$ Department of Rheumatology, Xuzhou Central Hospital, Xuzhou, China

${ }^{6}$ Department of Rheumatology, The Third Affiliated Hospital of Soochow University, Changzhou, China

${ }^{7}$ Department of Endocrinology, Yancheng Third People's Hospital, Yancheng, China

${ }^{8}$ Department of Rheumatology, Lianyungang Second People's Hospital, Lianyungang, China

${ }^{9}$ Department of Rheumatology, Lianyungang First People's Hospital, Lianyungang,

China

${ }^{10}$ Department of Rheumatology, Northern Jiangsu People's Hospital, Yangzhou, China

${ }^{11}$ Department of Rheumatology, Wuxi People's Hospital, Wuxi, China

${ }^{12}$ Department of Rheumatology, Jiangsu Province Hospital of Traditional Chinese

Medicine, Nanjing, China

${ }^{13}$ Department of Rheumatology, Southeast University Zhongda Hospital, Nanjing, China

${ }^{14}$ Department of Rheumatology, The First Affiliated Hospital of Soochow University, Suzhou, China

${ }^{15}$ Department of Rheumatology, Wuxi TCM Hospital, Wuxi, China

${ }^{16}$ Department of Rheumatology, Zhenjiang First People's Hospital, Zhenjiang, China

${ }^{17}$ Department of Rheumatology, Affiliated Hospital of Nantong University, Nantong, China

${ }^{18}$ Department of Rheumatology, Jiangsu Province Hospital, Nanjing, China

${ }^{19}$ Department of Rheumatology, Affiliated Hospital of Jiangsu University, Zhenjiang, China

Correspondence to Professor Lingyun Sun; lingyunsun@nju.edu.cn and Dr Xuebing Feng, Department of Rheumatology and Immunology, The Affiliated Drum Tower Hospital of Nanjing University Medical School, Nanjing 210008, China; fengxuebing@hotmail.com

Acknowledgements The authors thank all those members of Jiangsu Lupus Collaborative Group who followed up the patients and helped data collection. They acknowledge the Cinkate Corp for helping build and manage their lupus database.

Contributors FW and WZ collected data, analysed results and FW wrote the manuscript. SW, WP, LL, MW, FD, HH, XD, HW, YZ, XQ, MW, JW, JTao, JTan, ZD, $\mathrm{MZ}$ and $\mathrm{JL}$ acquired clinical data. LS and XF designed the study. XF edited the manuscript. LS supervised the project. All authors made substantial intellectual contributions to conception of the work, the interpretation of data and approval of the final manuscript.
Funding This study was funded by National Natural Science Foundation of China (grant no: 81302559, 81373198), Nanjing Medical Science and Technique Development Foundation (grant no: QRX17122), Jiangsu Provincial Special Program of Medical Science (grant no: BE2015602).

Competing interests None declared.

Patient consent Not required.

Provenance and peer review Not commissioned; internally peer reviewed.

(c) Article author(s) (or their employer(s) unless otherwise stated in the text of the article) 2019. All rights reserved. No commercial use is permitted unless otherwise expressly granted.

FW and WZ contributed equally.

\section{Check for updates}

To cite Wang F, Zhang W, Wang S, et al. Ann Rheum Dis 2019;78:e80.

Received 24 May 2018

Revised 29 May 2018

Accepted 31 May 2018

Published Online First 26 June 2018

\section{SLinked}

- http://dx.doi.org/10.1136/annrheumdis-2018-213853

Ann Rheum Dis 2019;78:e80. doi:10.1136/annrheumdis-2018-213819

\section{REFERENCES}

1 Müller-Calleja N, Manukyan D, Canisius A, et al. Hydroxychloroquine inhibits proinflammatory signalling pathways by targeting endosomal NADPH oxidase. Ann Rheum Dis 2017;76:891-7.

2 Rahman P, Gladman DD, Urowitz MB, et al. The cholesterol lowering effect of antimalarial drugs is enhanced in patients with lupus taking corticosteroid drugs. J Rheumatol 1999:26:325-30.

3 Petri M. Hydroxychloroquine use in the Baltimore Lupus Cohort: effects on lipids, glucose and thrombosis. Lupus 1996;5(Suppl 1):S16-22.

4 Ho KT, Ahn CW, Alarcón GS, et al. Systemic lupus erythematosus in a multiethnic cohort (LUMINA): XXVIII. Factors predictive of thrombotic events. Rheumatology 2005:44:1303-7.

5 Feng X, Pan W, Liu L, et al. Prognosis for hospitalized patients with systemic lupus erythematosus in China: 5-year update of the jiangsu cohort. PLoS One 2016;11:e0168619.

6 Alarcón GS, McGwin G, Bertoli AM, et al. Effect of hydroxychloroquine on the survival of patients with systemic lupus erythematosus: data from LUMINA, a multiethnic US cohort (LUMINA L). Ann Rheum Dis 2007;66:1168-72.

7 Shinjo SK, Bonfá E, Wojdyla D, et al. Antimalarial treatment may have a timedependent effect on lupus survival: data from a multinational Latin American inception cohort. Arthritis Rheum 2010;62:855-62.

8 Costedoat-Chalumeau N, Galicier L, Aumaître 0, et al. Hydroxychloroquine in systemic lupus erythematosus: results of a French multicentre controlled trial (PLUS Study). Ann Rheum Dis 2013:72:1786-92.

9 Pons-Estel GJ, Alarcón GS, McGwin G, et al. Protective effect of hydroxychloroquine on renal damage in patients with lupus nephritis: LXV, data from a multiethnic US cohort. Arthritis Rheum 2009;61:830-9. 